\title{
Motion of discrete solitons assisted by nonlinearity management
}

\author{
Jesús Cuevas, ${ }^{1}$ Boris A. Malomed, ${ }^{2}$ and P. G. Kevrekidis ${ }^{3}$ \\ ${ }^{1}$ Grupo de Física No Lineal, Departamento de Física Aplicada I, Escuela Universitaria Politécnica, \\ C/ Virgen de África, 7, 41011 Sevilla, Spain \\ ${ }^{2}$ Department of Interdisciplinary Studies, School of Electrical Engineering, Faculty of Engineering, Tel Aviv University, \\ Tel Aviv 69978, Israel \\ ${ }^{3}$ Department of Mathematics and Statistics, University of Massachusetts, Amherst, Massachusetts 01003-4515, USA
}

(Received 12 November 2004; published 29 June 2005)

\begin{abstract}
We demonstrate that time-periodic modulation of the nonlinearity coefficient in the discrete nonlinear Schrödinger equation strongly facilitates creation of traveling solitons in the lattice. We predict this possibility in a semiqualitative form analytically, and test it in direct numerical simulations. Systematic computations reveal several generic dynamical regimes, depending on the amplitude and frequency of the time modulation, and on the initial thrust which sets the soliton in motion. These regimes include irregular motion of the soliton, regular motion of a decaying one, and regular motion of a stable soliton. The motion may occur in both the straight and reverse directions, relative to the initial thrust. In the case of stable motion, extremely long simulations in a lattice with periodic boundary conditions demonstrate that the soliton keeps moving indefinitely long without any visible loss. Velocities of moving stable solitons are in good agreement with the analytical prediction, which is based on requiring a resonance between the ac drive and motion of the soliton through the periodic lattice. The generic dynamical regimes are mapped in the model's parameter space. Collisions between moving stable solitons are briefly investigated too, with a conclusion that two different outcomes are possible: elastic bounce, or bounce with mass transfer from one soliton to the other. The model can be realized experimentally in a Bose-Einstein condensate trapped in a deep optical lattice.
\end{abstract}

DOI: 10.1103/PhysRevE.71.066614 PACS number(s): 05.45.Yv, 42.65.Wi, 05.45.-a, 03.75.Lm

\section{INTRODUCTION}

The discrete nonlinear Schrödinger (DNLS) equation is a well-known model of nonlinear lattice dynamics, which allows to study many generic features of nonintegrable dynamics [1]. This equation also finds direct applications to arrays of nonlinear optical waveguides (as it was predicted long ago [2] and demonstrated in detail more recently, see Refs. [3] and references therein), and to arrays of droplets in BoseEinstein condensates (BECs) trapped in a very deep optical lattice [4].

In all these contexts, discrete solitons are fundamental dynamical excitations supported by the DNLS equation. The dynamics of standing solitons, pinned by the underlying lattice, is understood quite well, in terms of both numerical simulations and analytical approximations, the most general one being based on the variational method [5]. However, moving discrete solitons pose a much more complex issue $[6,7]$. While, strictly speaking, exact solutions for moving solitons should not exist because of the radiation loss, direct simulations indicate that a soliton may move freely if its "mass" (quadratic norm) does not exceed a certain critical value [7]. In the quasicontinuum approximation, the source of the braking force acting on the moving soliton is the effective Peierls-Nabarro (PN) potential induced by the lattice [8].

In the case of the DNLS equation describing an array of nearly isolated droplets of a BEC in a deep optical lattice, an interesting possibility is to apply the ac Feshbach-resonance management (FRM) to it, as it was recently proposed in Ref. [9]. FRM may be induced by an external ac magnetic field, which periodically (in time) changes the sign of the nonlin- earity by dint of the Feshbach resonance affecting collisions between atoms (for a one-dimensional BEC without the optical lattice, the concept of FRM was elaborated in Ref. [10]).

In this work, our aim is to demonstrate that the FRM, applied to the DNLS model, can strongly facilitate the motion of discrete solitons, which is a notable dynamical effect in the discrete systems (Ref. [9] was only dealing with standing ones). The corresponding DNLS equation is

$$
i \dot{u}_{n}+u_{n+1}+u_{n-1}-2 u_{n}+g(t)\left|u_{n}\right|^{2} u_{n}=0,
$$

where $u_{n}(t)$ are the BEC wave functions at the lattice sites, and the real time-dependent nonlinear coefficient, proportional to the scattering length of the interatomic collisions (with the sign minus), is

$$
g(t)=g_{\mathrm{dc}}+g_{\mathrm{ac}} \sin (\omega t) .
$$

Note that $g_{\text {dc }}$ can be always chosen positive, because Eq. (1) with $g_{\mathrm{dc}}<0$ can be transformed into that with $g_{\mathrm{dc}}>0$ by means of the so-called staggering transformation

$$
u_{n}(t) \equiv(-1)^{n} e^{-4 i t} \widetilde{u}_{n}(t)
$$

The subsequent presentation is structured as follows: In Sec. II, using a Gaussian approximation for the soliton, we present an analytical estimate of the effective PN potential for the moving discrete soliton. The estimate suggests that the ac modulation of $g(t)$ may indeed help to suppress the PN potential, and thus facilitate free motion of discrete solitons. In Sec. III, we display results of systematic simulations, summarized in the form of diagrams in the parameter plane 
$\left(\omega, g_{\text {ac }}\right)$. The diagrams feature several generic dynamical regimes, including a large area of stable progressive motion. Collisions between solitons moving in opposite directions are briefly considered too, with a conclusion that they bounce from each other, sometimes featuring mass transfer between the solitons. Diverse dynamical regimes predicted in this work suggest straightforward possibilities for new experiments in BECs trapped in deep optical lattices, as discussed in the concluding Sec. IV which summarizes the findings reported in this paper.

\section{ANALYTICAL APPROXIMATION}

The continuum limit suggests the following ansatz for a moving soliton [11],

$$
\begin{aligned}
u_{\mathrm{ans}}(n, t)= & A \exp \left(-a[n-\xi(t)]^{2}+i \phi(t)+(i / 2) \dot{\xi} n\right. \\
& \left.-(i / 4) \int[\dot{\xi}(t)]^{2} d t\right),
\end{aligned}
$$

where $A, a, \xi(t)$, and $\phi$ are, respectively, the amplitude, squared inverse width, center position, and phase of the soliton. Accordingly, $\dot{\xi}$ is the soliton's velocity, $\dot{\xi} / 2$ simultaneously being the wave number of the wave field carrying the moving soliton. For the soliton in the continuum NLS equation, the variational approximation yields the following relations:

$$
\dot{\phi}=3 a, \quad A^{2}=4 \sqrt{2} a / g,
$$

if $g=$ const $>0$. In Eqs. (5), $a$ is regarded as an arbitrary positive constant (intrinsic parameter of the soliton family).

The only approximation which can produce a tractable result assumes a quasicontinuum ansatz for the soliton in the discrete system. Following this approach, we use the Hamiltonian corresponding to the DNLS equation (1):

$$
H=\sum_{n=-\infty}^{+\infty}\left(2\left|u_{n}\right|^{2}-\left(u_{n}^{*} u_{n+1}+u_{n} u_{n+1}^{*}\right)-\frac{g}{2}\left|u_{n}\right|^{4}\right),
$$

the asterisk standing for the complex conjugation, and substituting into it the ansatz (4). This way, the potential of the soliton-lattice interaction is obtained in the form of a Fourier series, $H(\xi, \dot{\xi})=\sum_{m=0}^{\infty} H_{m}(\dot{\xi}) \cos (2 \pi m \xi)$. In the case of a broad soliton, for which the ansatz (4) is relevant (or, in a more general case, just to have the approximation in a tractable form), we keep only the lowest harmonic $(m=1)$ in this expression, which is nothing else but the PN potential $U_{\mathrm{PN}}$. After some straightforward algebra (using the Poisson summation formula), we thus find

$$
\begin{aligned}
U_{\mathrm{PN}}(\xi, \dot{\xi})= & \frac{1}{2} \sqrt{\frac{\pi}{a}} A^{2} \exp \left(-\frac{\pi^{2}}{4 a}\right)\left[4 \sqrt{2} \exp \left(-\frac{\pi^{2}}{4 a}\right)\right. \\
& \left.\times\left[1+e^{-a / 2} \cos (\dot{\xi} / 2)\right]-g A^{2} \sqrt{\frac{\pi}{a}}\right] \cos (2 \pi \xi) .
\end{aligned}
$$

A noteworthy feature in this estimate, in comparison with
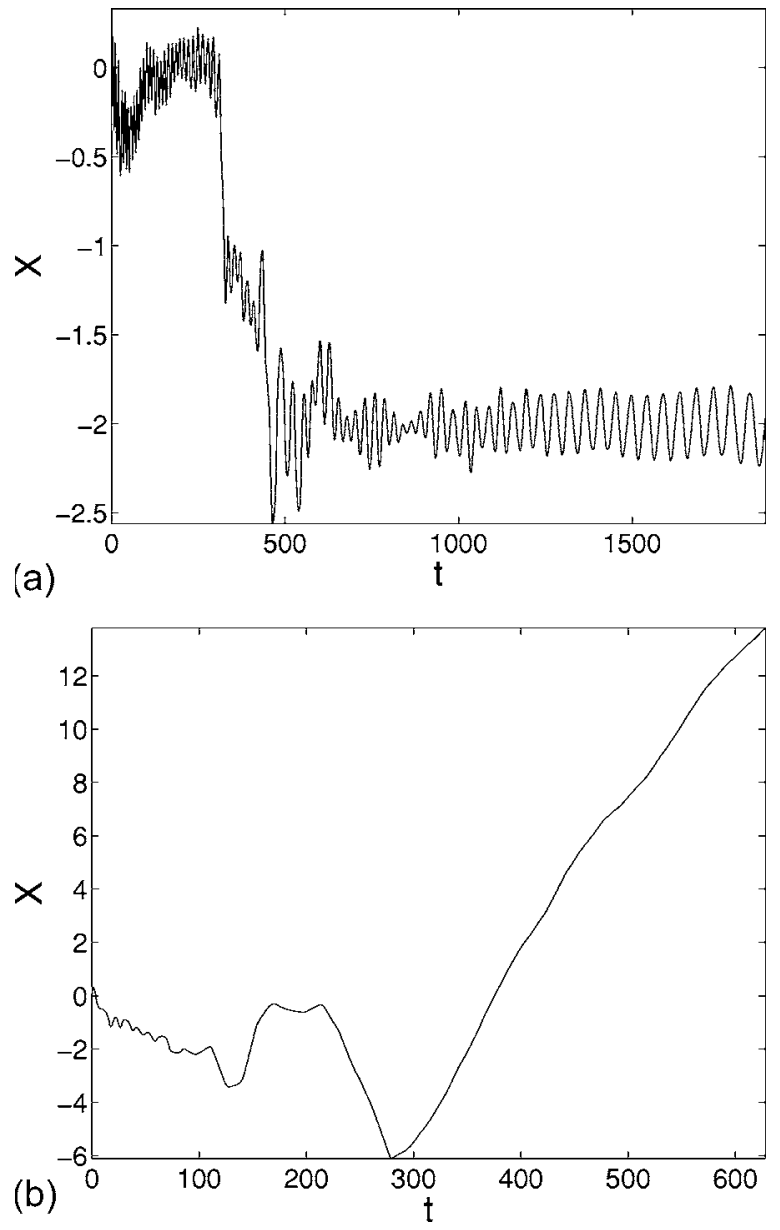

FIG. 1. Position of the center of mass $X$ as a function of time for a typical example of a dynamical regime in which (a) the soliton remains pinned $\left(g_{\mathrm{ac}}=0.03, \omega=1\right.$, and $\left.q=0.5\right)$ and (b) the soliton develops an irregular motion $\left(g_{\mathrm{ac}}=0.065, \omega=1\right.$, and $\left.q=0.5\right)$.

known perturbative results for static solitons [8], is the dependence on the potential's amplitude on the soliton's velocity, $\dot{\xi}$.

If the relation between $a$ and $A^{2}$ for the soliton in the continuum NLS equation, as given by Eq. (5)(for constant $g$ ), is substituted in Eq. (7), the coefficient in front of $\cos (2 \pi \xi)$, i.e., the amplitude of the PN potential, never vanishes. However, it may vanish if the underlying pulse (4) is considered not as a soliton, but just as a pulse with independent amplitude and inverse-width parameters $A^{2}$ and $a$; then, the condition of the vanishing of the PN potential determines a discrete spectrum of the velocities $\dot{\xi}$, in the form

$$
1+\exp (-a / 2) \cos (\dot{\xi} / 2)=\left(g A^{2} / 4\right) \sqrt{\pi /(2 a)} \exp \left[\pi^{2} /(4 a)\right],
$$

provided that $g A^{2}$ is small enough to make the right-hand side of Eq. (8) smaller than 2 (this caveat is essential, as the factor $\exp \left[\pi^{2} /(4 a)\right]$ may be exponentially large).

In this work, however, our objective is not to verify this possibility, but rather to consider the case when the nonlinear coefficient $g$ is a function of time, as defined in Eq. (2). Note 

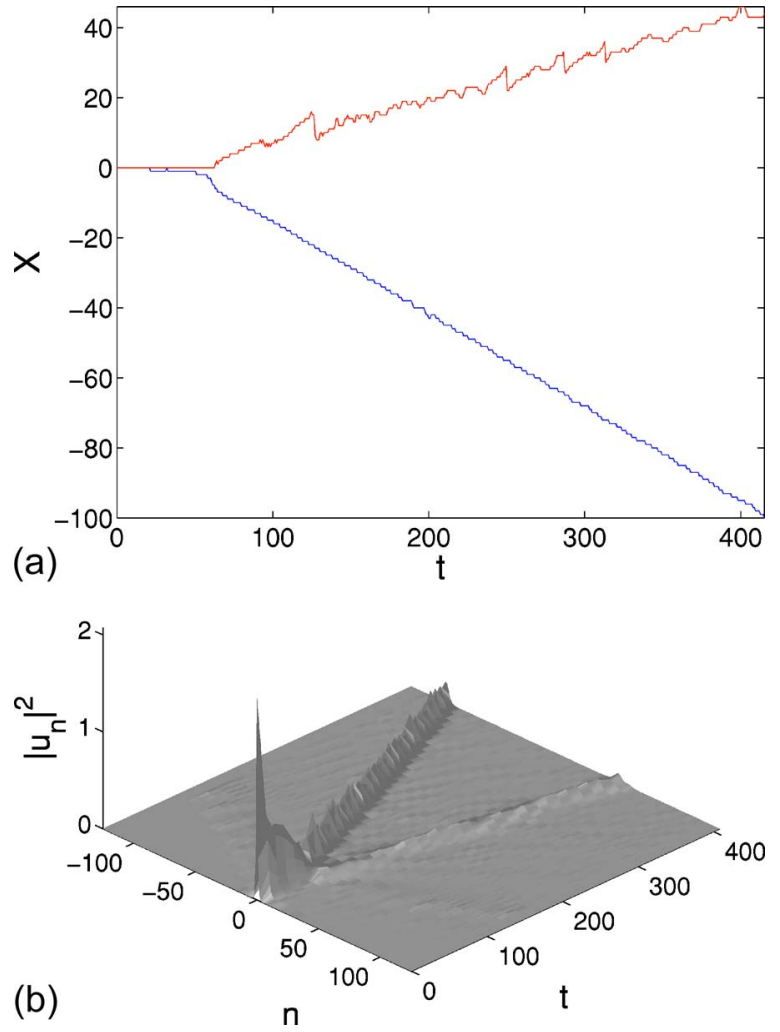

FIG. 2. An example of asymmetric splitting of the soliton, for $g_{\text {ac }}=0.196, \omega=0.5$, and $q=0.5$. Panel (a) shows the position of two local density maxima corresponding to the secondary solitons (splinters), and panel (b) shows the global evolution of the lattice field.

that, for a broad soliton ( small $a$ ), the PN potential barrier is exponentially small, hence the soliton's kinetic energy may be much larger than the potential. This implies that the velocity of the soliton moving through the potential (7) with the period $L=1$ contains a constant (dc) part and a small ac correction to it, with the frequency $2 \pi \dot{\xi}_{0} / L \equiv 2 \pi \dot{\xi}_{0}[12]$,

$$
\dot{\xi}(t) \approx \dot{\xi}_{0}+\dot{\xi}_{1} \cos \left(2 \pi \dot{\xi}_{0} t\right), \quad \dot{\xi}_{1}^{2} \ll \dot{\xi}_{0}^{2}
$$

Then, substituting the expression (9) into the condition (8), which provides for the suppression of the PN potential, one can expand its left-hand side,

$$
\begin{aligned}
1+\exp (-a / 2) \cos (\dot{\xi} / 2) \approx & 1+\exp (-a / 2)\left[\cos \left(\dot{\xi}_{0} / 2\right)\right. \\
& \left.-\left(\dot{\xi}_{1} / 2\right) \sin \left(\dot{\xi}_{0} / 2\right) \cos \left(2 \pi \dot{\xi}_{0} t\right)\right]
\end{aligned}
$$

Now, inserting the variable $g(t)$ from Eq. (2) into the righthand side of Eq. (8), it is obvious that $g_{\text {dc }}$ and $g_{\text {ac }}$ can be chosen so as to secure this equation to hold, provided that the average soliton's velocity takes the resonant value, $\dot{\xi}_{0}$ $=\omega / 2 \pi$. More generally, due to anharmonic effects, one may expect the existence of a spectrum of the resonant velocities,
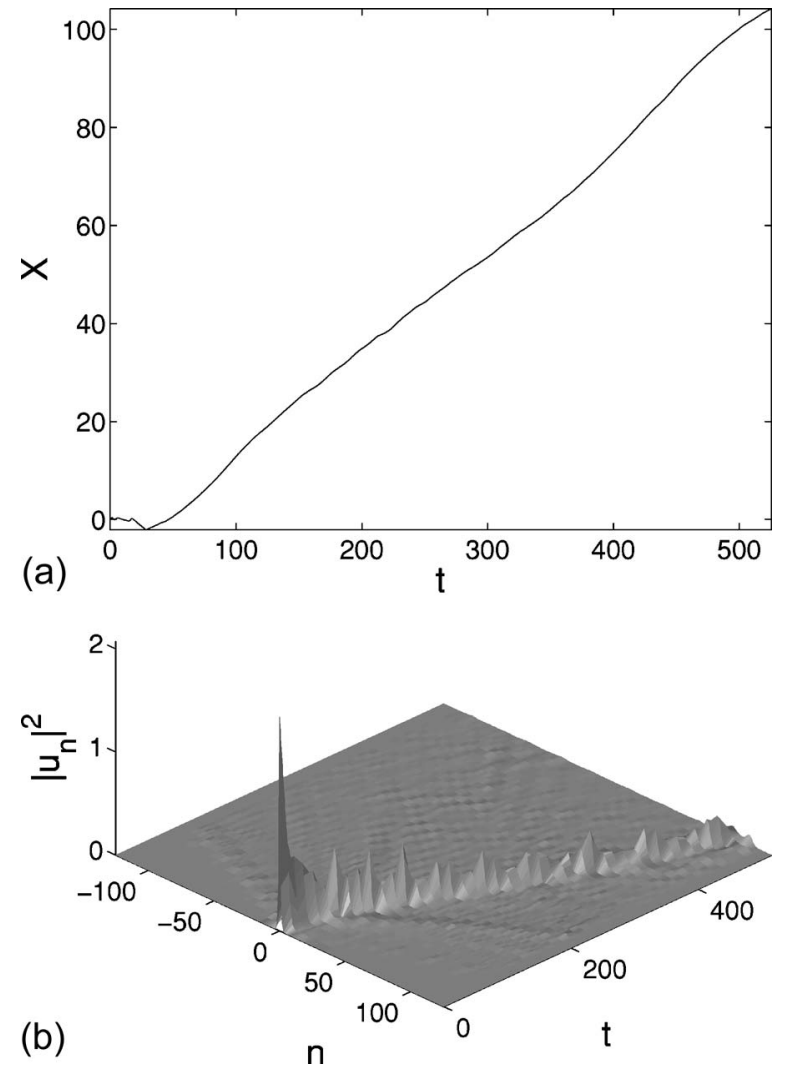

FIG. 3. A generic example of the progressive motion of a decaying soliton in the straight direction, for $g_{\text {ac }}=0.206, \omega=0.5$, and $q=0.5$.

$$
\dot{\xi}_{0}=\left(c_{\text {res }}\right)_{N}^{(M)} \equiv \frac{M \omega}{2 \pi N},
$$

with integers $M$ and $N$.

Actually, an ac drive can support stable progressive motion of solitons at the resonant velocities (11) (assuming the spatial period $L=1$ ), even in the presence of dissipation, in a broad class of systems. This effect was first predicted for discrete systems (of the Toda-lattice and Frenkel-Kontorova types) in Ref. [13], and demonstrated experimentally in an LC electric transmission line in Ref. [14]. Later, the same effect was predicted [15] and demonstrated experimentally [16] in continuous long Josephson junctions with a spatially periodic inhomogeneity. However, a qualitative difference of the present prediction is that it applies to nontopological solitons, while all the previously known examples involved kinks, i.e., discrete of continuum solitons whose topological charge directly couples to the driving field [this is why the above approximation, even if it is a crude one, is relevant, as the relation (11), established for the ac-driven kinks, cannot be immediately applied to nontopological solitons]. The only example similar to what is suggested here in the context of nontopological solitons that we are aware of, was reported in Ref. [17] (the model involved spatially uniform dissipation and an ac drive localized in space). Note also that the mechanism of the ac-driven motion considered here is different from that in ratchet systems (see, e.g., Ref. [18] and refer- 

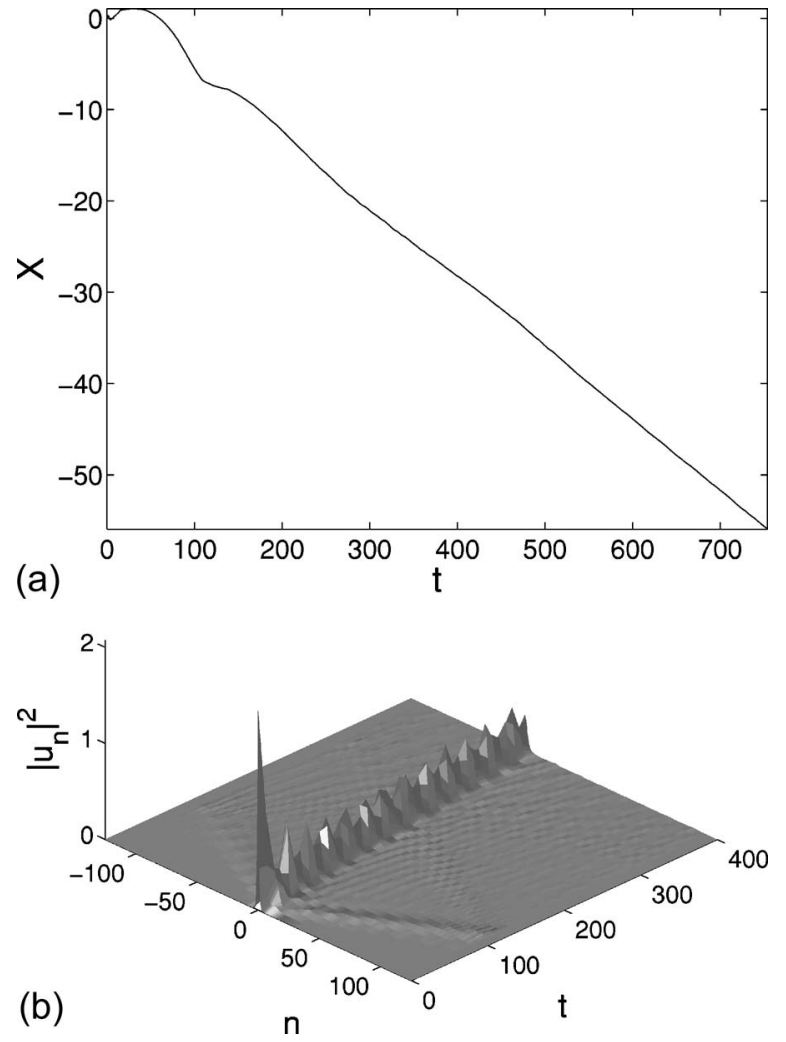

FIG. 4. The same as in Fig. 4, in the case of motion in the reverse direction, for $g_{\mathrm{ac}}=0.170, \omega=1$, and $q=0.5$.

ences therein), as the DNLS equation does not break the mirror symmetry, $n \rightleftarrows-n$.

\section{NUMERICAL RESULTS}

We now proceed to direct simulations of the system discussing some of the relevant theoretical predictions in light of the numerical results. We integrate Eq. (1) with an initial configuration in the form of a standing-soliton solution for $g_{\mathrm{ac}}=0$. This solution has the form of $u_{n}(t)=v_{n} \exp (i \chi t)$, with the real field $v_{n}$ obeying the equation

$$
\chi v_{n}=v_{n+1}+v_{n-1}-2 v_{n}+g_{\mathrm{dc}} v_{n}^{3} .
$$

Equation (12) was solved by means of well-known methods (starting from the anticontinuum limit). Then, to set the soliton in motion, it was given a lattice momentum $q$, so that the initial condition was

$$
u_{n}(0)=v_{n} \exp (\text { inq } / 2) .
$$

Equation (13) implies that the soliton will move to the right if $q>0$.

The results will be displayed for $g_{\mathrm{dc}}=1, \chi=1$, and three different values of the initial thrust, $q=0.25, q=0.5$, and $q$ $=1$, as these cases were found to represent a generic situation in the plane of the ac-drive's parameters $\left(\omega, g_{\text {ac }}\right)$. Simulations were run in the time interval $0<t<100 \times(2 \pi / \omega)$ or longer, by means of the fourth-order Runge-Kutta algorithm, with the time step $\Delta t=0.002$. In most cases, the lattice with 251 sites was used. Edge absorbers were installed by adding the
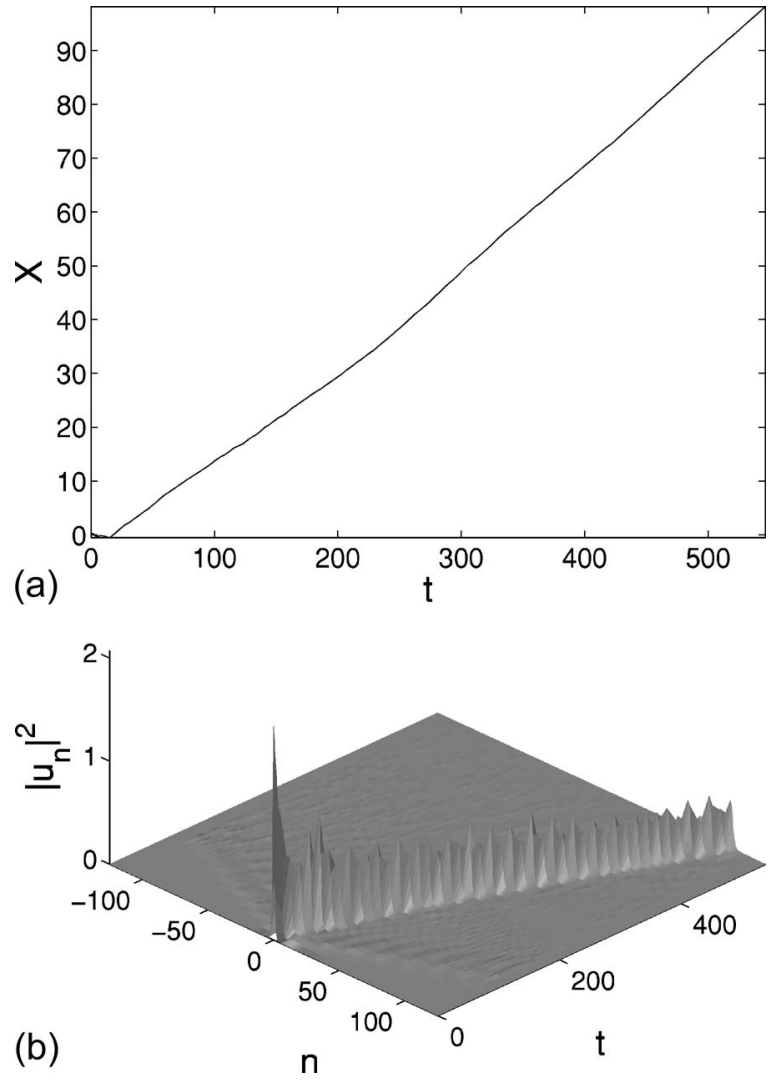

FIG. 5. A generic example of the motion of a stable (nondecaying) soliton in the straight direction, for $g_{\mathrm{ac}}=0.132, \omega=1$, and $q=0.5$.

loss term $i \gamma u_{n}$, with $\gamma=1$, to Eq. (1) at the 10 sites adjacent to each edge. Besides that, in some cases extremely long simulations were performed in a larger lattice with periodic boundary conditions, to verify if the stable motion of the soliton could last for very long times, and also to examine collisions between the solitons, see below.

If $g_{\mathrm{ac}}=0$, the soliton pushed as per Eq. (13) with $q \lesssim 0.7$ does not start progressive motion. Instead, it remains pinned to the lattice, with its center oscillating around an equilibrium position. This observation may be explained by the fact that the kinetic energy given to the soliton is smaller than the height of the PN potential barrier.

Several distinct types of dynamics were observed with $g_{\text {ac }}>0$, depending on the driving frequency $\omega$ and the thrust parameter $q$. First, the soliton may remain pinned, as shown in Fig. 1(a). In this case, the simulations [run in the interval $0<t<300(2 \pi / \omega)]$ demonstrate that the soliton stays pinned within a few sites from its initial position. The central coordinate, the evolution of which is displayed in Fig. 1 and other figures, is defined as

$$
X=\frac{\sum_{n} n\left|u_{n}\right|^{2}}{\sum_{n}\left|u_{n}\right|^{2}} .
$$

The next generic regime is that of irregular motion, as shown in Fig. 1(b). A characteristic feature of this regime is 

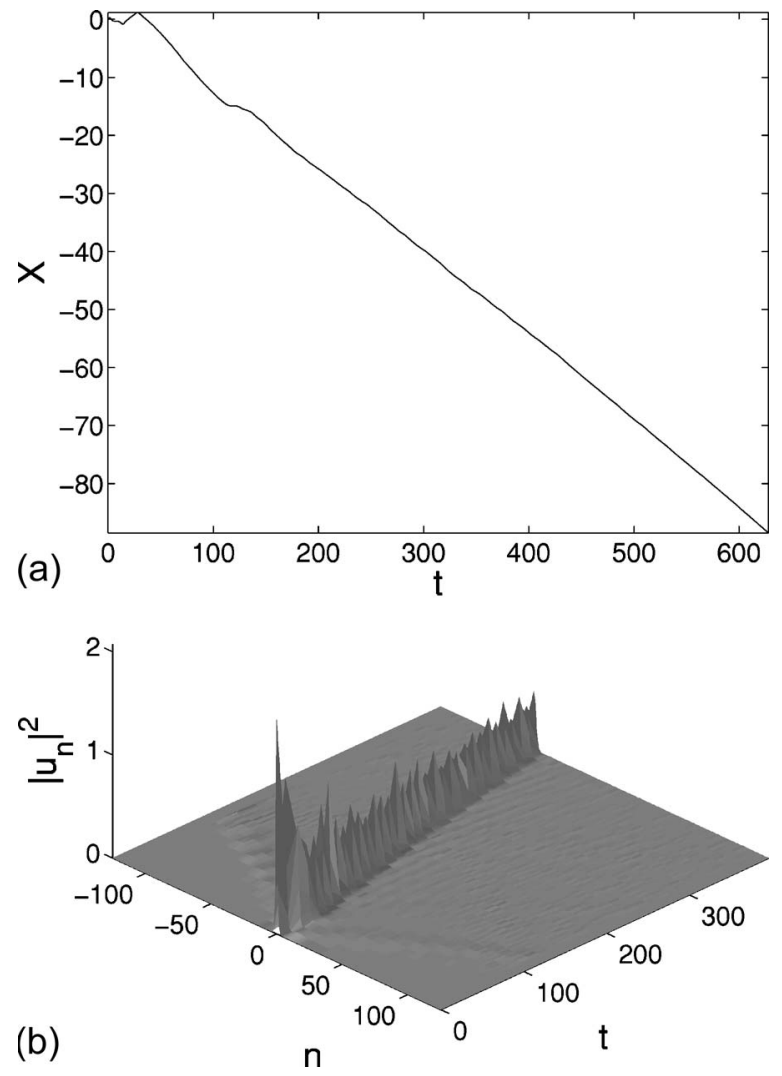

FIG. 6. The same as in Fig. 6, but in the case of the motion of a stable soliton in the reverse direction, for $g_{\mathrm{ac}}=0.122, \omega=1$, and $q=0.5$.

that the soliton randomly changes the direction of motion several times, and the velocity remains very small in comparison with regimes of "true motion," see below.

Under the action of a strong drive, the soliton can sometimes split into two secondary ones moving in opposite directions, see Fig. 2. As can be observed, the splitting is strongly asymmetric, and the heavier secondary soliton (splinter) may move both forward and backward, relative to the initial push. We notice that splitting of a quiescent soliton (without any initial thrust applied to it) into two symmetric splinters, moving in opposite directions with equal velocities, was reported, in the same model based on Eq. (1), in Ref. [9]. The initial push applied to the soliton is a natural cause for the symmetry breaking observed here in the case of the splitting.

In the case of a moderately strong drive, the splitting does not occur (see Fig. 9 below). Instead, two distinct generic regimes of regular motion of the soliton were observed. In one case, shown in Figs. 3 and 4, the moving soliton is not really stable, as it gradually decays into radiation. The most interesting case is that of persistent motion of a stable soliton, without any observable decay (after an initial transient stage of the evolution). Examples of the latter are displayed in Figs. 5 and 6. To distinguish between the two different regimes of motion, we have adopted a criterion that the moving soliton is stable if it keeps more than $70 \%$ of its norm, $\Sigma\left|u_{n}\right|^{2}$, in the core. It was found that the criterion in this form makes it possible to accurately identify truly stable regimes,
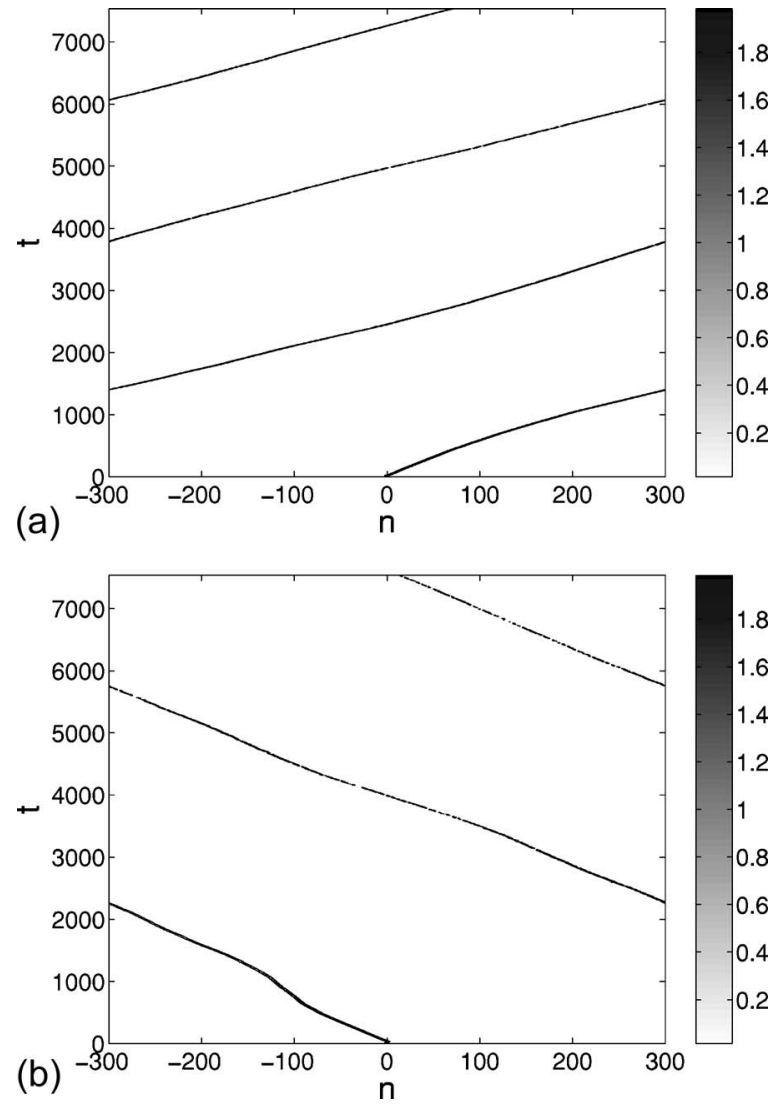

FIG. 7. Indefinitely long motion of stable solitons in a ring lattice composed of 601 sites. The gray-scale plots show the spatiotemporal distribution of the density, $\left|u_{n}(t)\right|^{2}$. The examples of the straight (a) and inverse (b) motion pertain, respectively, to $g_{\text {ac }}=0.132, \omega=1, q=0.5$, and $g_{\text {ac }}=0.122, \omega=1, q=0.5$.

if the simulation is extended for a much longer time (see below). Typical examples displayed in Figs. 3-6 demonstrate that free motion of the soliton is possible in both the straight and reverse directions, relative to the initial thrust.

As stable motion of solitons in nonintegrable discrete models is an issue of theoretical and experimental interest, we have further investigated this case, replacing the finite lattice with edge absorbers by a ring-shaped one, with periodic boundary conditions. This setting opens a way to study indefinitely long motion of the soliton. The result, illustrated by examples shown in Fig. 7, is that the moving solitons which were identified as stable by the above criterion, remain stable indeed (preserving their shape) as long as the simulations could be run. In this case, it is pertinent to compare the average velocity $\bar{c}$ of the persistent motion with the prediction given by Eq. (11). The result is $\bar{c}_{1} \approx 0.246$ and $\bar{c}_{2} \approx 0.155$ in the cases shown in Figs. $7(\mathrm{a})$ and $7(\mathrm{~b})$, respectively. Comparison with the analytical formula (11) (with $\omega=1$, which is the driving frequency in the examples shown in Fig. 7) demonstrates that $\bar{c}_{2}$ and $\bar{c}_{1}$ fit well to the predicted values in the cases of the, respectively, fundamental and second-order resonance,

$$
\bar{c}_{2} /\left(c_{\text {res }}\right)_{1}^{(1)} \approx 0.974, \quad \bar{c}_{1} /\left(c_{\text {res }}\right)_{3}^{(2)} \approx 1.029 .
$$

Relatively small discrepancies between the predicted and observed values in Eq. (15) can be accounted for by the fact 

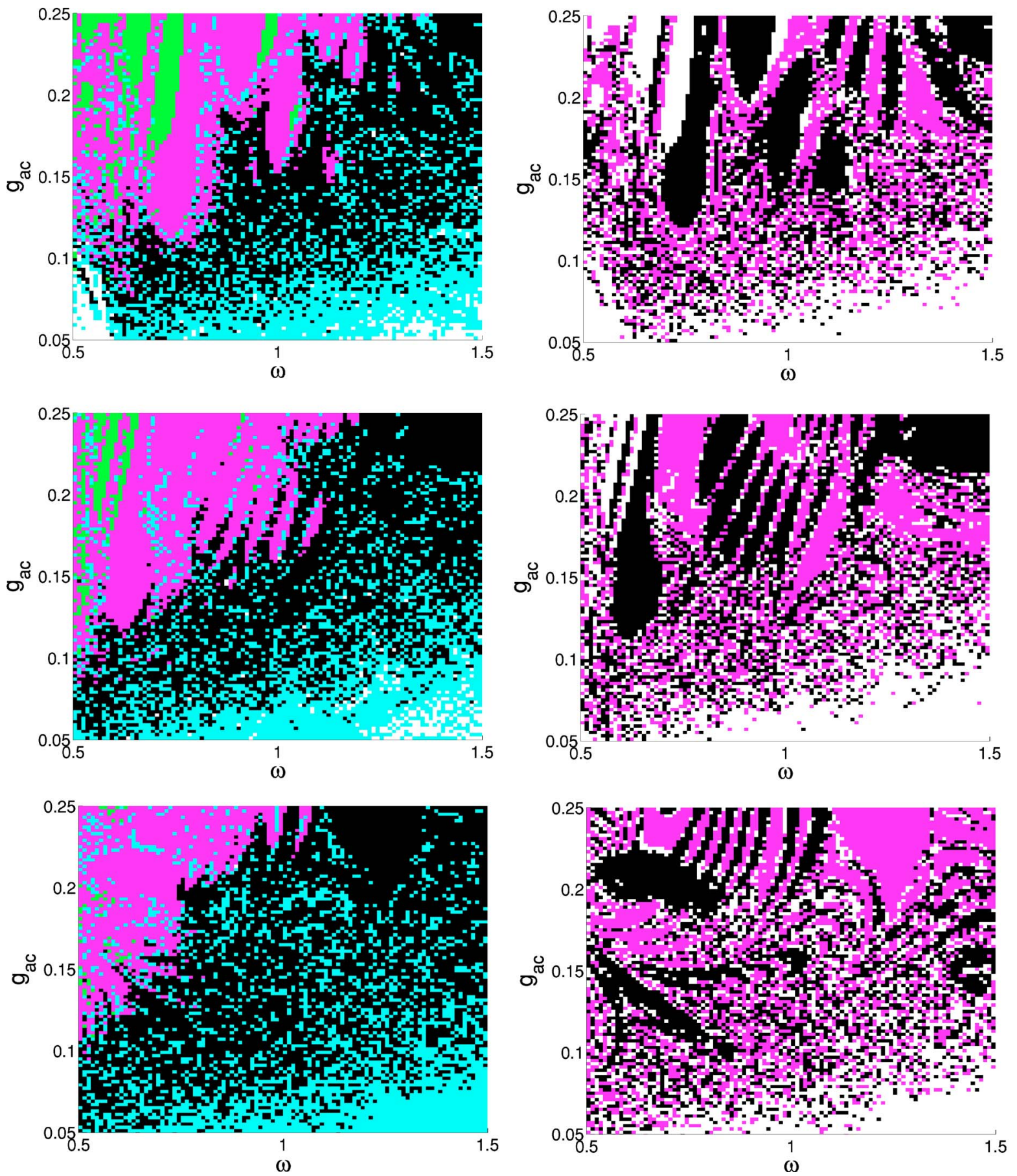

FIG. 8. (Color online) Maps in the left column show areas in the parameter plane $\left(\omega, g_{\text {ac }}\right)$ which give rise to the following dynamical regimes. The rows from top to bottom correspond to values $0.25,0.5$, and 1 of the initial-push parameter $q$. White areas: the soliton remains pinned; cyan: irregular motion; green: splitting; magenta: regular motion with decay; black: stable motion (without decay). The maps in the right column additionally show the difference between the forward (alias straight, marked by magenta) and backward (alias reverse, marked by black) directions of the regular motion, relative to the direction singled out by the initial push. Regular-motion regimes for both decaying and stable solitons are included here.

that the effective perturbations are not really weak in these cases.

The soliton adjusting itself to the stable-motion mode typically sheds off about $20 \%$ of its initial norm. This conspicuous amount of radiation does not essentially affect the established regime. In the cases shown in Figs. 3-6 the emitted radiation is partly suppressed by the edge absorbers.
However, even in the case displayed in Fig. 7, when all the radiation stays in the lattice subject to the periodic boundary conditions, its presence does not give rise to any appreciable perturbation in the long-time motion of the soliton. In fact, this observation is an additional essential evidence of the robustness of the moving soliton in the corresponding regime. 
For the fixed values of $g_{\mathrm{dc}}=1$ in Eq. (2) and $\chi=1$ in Eq. (13), and several values of the initial thrust in Eq. (13), $q=0.25,0.5$, and 1 , we have collected results of systematic simulations, varying the drive's parameters, $g_{\text {ac }}$ and $\omega$, by small steps in broad ranges spanning the two-parameter space. The results are summarized in Fig. 8, in the form of maps in the $\left(\omega, g_{\mathrm{ac}}\right)$ plane, where we outline regions giving rise to each of the qualitatively different dynamical regimes described above. This map is the key finding of the present work, detailing the various possibilities arising as a result of the application of the FRM to the lattice soliton.

Some general features can be deduced from the examination of the maps in Fig. 8. The increase of the initial thrust $q$ significantly affects the map, although chiefly quantitatively, rather than qualitatively. At all values of $q$, the irregular motion is, generally, changed by stable progressive motion (straight or reverse) with the increase of the drive's amplitude, and/or decrease of its frequency, which seems quite natural. Further increase of the drive's strength, which implies the applications of a strong perturbation to the system, may be expected to lead to an instability, which indeed happens, in the form of onset of the gradual decay of the moving solitons. Finally, strong instability sets in, manifesting itself in the splitting of the soliton. It also seems natural that the soliton is more prone to splitting if the driving frequency is low, as internal strain in the pulse, which eventually leads to its splitting, has more time to accumulate if the drive oscillates slowly.

Transition to the reverse motion tends to happen parallel to the transition from the stable moving soliton to the decaying one. For this reason, in most cases (but not always) backward-moving solitons are decaying ones. Finally, it should be noted that the increase of the initial thrust leads to overall stabilization of the soliton (somewhat counterintuitively), making the decay and splitting zones smaller.

Finally, using the large lattice with the periodic boundary conditions, we also simulated collisions between solitons originally moving with opposite velocities. Initial pulses were generated by applying the thrust $\pm q$ to two quiescent solitons. A systematic study of collisions is very difficult in the present model, cf. Ref. [7]. Nevertheless, we were able to identify two different types of the interaction, typical examples of which are shown in Fig. 9. In the case of Fig. 9(a), the solitons bounce back from each other elastically. Afterwards, one of the solitons spontaneously reverses its direction of motion, due to its interaction with the underlying lattice. Eventually, we observe a pair of weakly interacting solitons traveling for long times in the same direction.

In another case, Fig. 9(b), the solitons also bounce after the first collision; however, in this case the collision is inelastic, resulting in transfer of mass (norm) from one soliton to the other. Repeated collisions lead to additional transfer, and eventually the weak soliton almost disappears. It is relevant to note that, in contrast to what is known about collisions between moving solitons in the ordinary DNLS equation (the one with constant coefficients) [7], we have never observed merger of colliding solitons into a standing one.

\section{CONCLUSIONS}

In this work, we have investigated moving nontopological solitons in the DNLS equation with a periodically time-
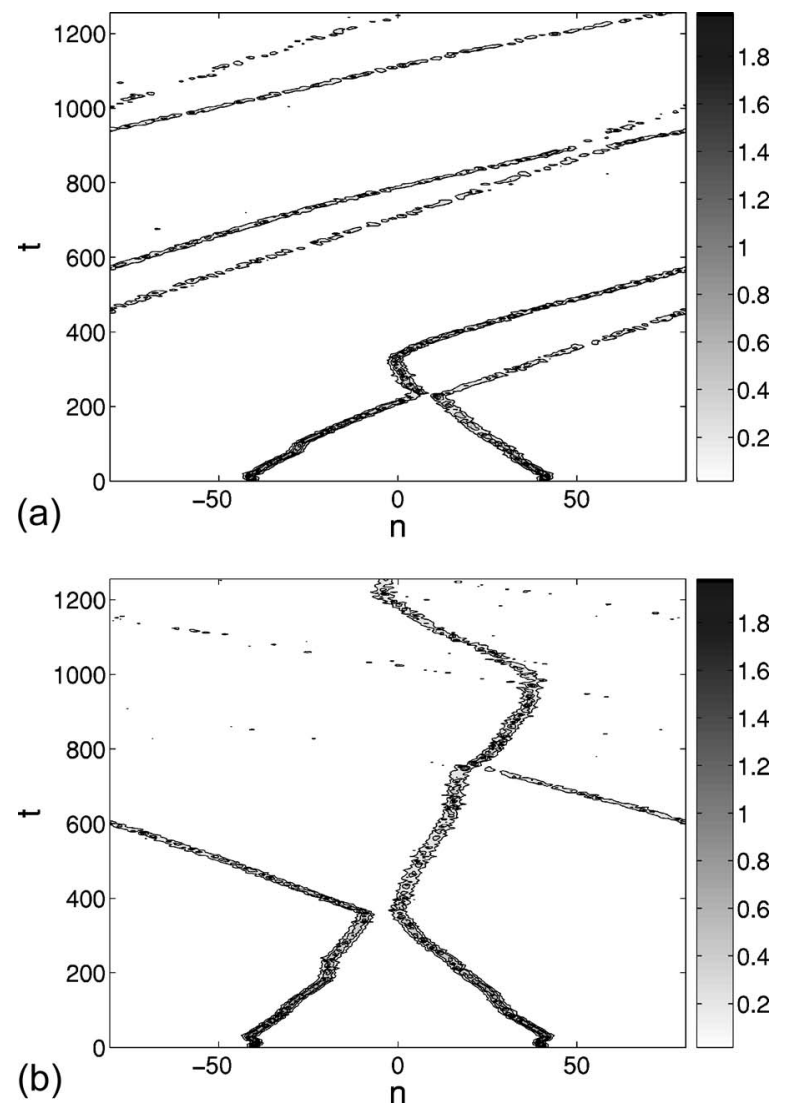

FIG. 9. Two typical examples of different outcomes of collisions between solitons with equal masses moving in opposite directions in the lattice with periodic boundary conditions. The parameters are $g_{\text {ac }}=0.132, \omega=1, q=0.5$ (a) and $g_{\text {ac }}=0.122, \omega=1, q=0.5$ (b). Note that the collision is multiple in panel (b).

modulated nonlinear coefficient. An approximate analytical consideration predicts that the ac nonlinearity management may support stable traveling solitons in the lattice. Systematic simulations reveal several generic dynamical regimes, depending on parameters of the time modulation, and the size of the initial thrust which sets the soliton in motion. Besides the possibility that the soliton remains pinned, or is split by a very strong drive, the basic dynamical regimes feature irregular motion, regular motion of a decaying soliton, and regular motion of a stable one. In the latter case, extremely long simulations in the lattice with periodic boundary conditions demonstrate that the soliton keeps moving indefinitely long without any tangible loss. Velocities of the moving stable solitons are found to be in good agreement with the analytical prediction through a resonance condition, a noteworthy fact being that the spectrum of the resonant velocities for the nontopological solitons is the same as for topological ones (kinks), although the dynamical mechanisms supporting the progressive motion in the two cases are quite different. All the generic dynamical regimes were mapped in the model's parameter space. Collisions between stable moving solitons were briefly investigated too, with a conclusion that two different outcomes are possible, elastic bounce, and bounce with mass transfer between the solitons.

The model can be implemented in a quasi-onedimensional ("cigar-shaped") Bose-Einstein condensate seg- 
mented by a strong optical lattice, the nonlinearity modulation induced by the Feshbach resonance in the ac regime. In this case, a realization is possible with $\sim 10^{3}$ atoms trapped in each of a few filled local potential wells. The setting may be essentially the same as in the recent experimental work which has resulted in the creation of one-dimensional gap solitons [19] and observation of the nonlinear self-trapping [20] in the repulsive condensate (without the Feshbach management). The fact that the repulsive condensate $\left({ }^{87} \mathrm{Rb}\right)$ was used in the experiments is not an obstacle, in view of the applicability of the staggering transformation of Eq. (3). Then, the moving soliton can be understood, physically, as a coherently self-translating wave that macroscopically tunnels between wells. In this respect, experimental observation of such a soliton would be a very interesting physical result.
In a more general setting, it would be interesting to examine similar dynamical scenarios for the effect of FRM on dark solitons, and, especially, to understand how this phenomenology is modified in higher dimensions. Such studies are currently in progress and will be reported in future publications.

\section{ACKNOWLEDGMENTS}

This work was partially supported by NSF-DMS0204585, NSF-CAREER, and the Eppley Foundation for Research (P.G.K.), the Israel Science Foundation Grant No. 8006/03 (B.A.M.), and the MECD/FEDER project FIS200401183 (J.C.).
[1] P. G. Kevrekidis, K. Ø. Rasmussen, and A. R. Bishop, Int. J. Mod. Phys. B 15, 2833 (2001).

[2] D. N. Christodoulides and R. I. Joseph, Opt. Lett. 13, 794 (1988).

[3] U. Peschel, R. Morandotti, J. M. Arnold, J. S. Aitchison, H. S. Eisenberg, Y. Silberberg, T. Pertsch, and F. Lederer, J. Opt. Soc. Am. B 19, 2637 (2002); H. S. Eisenberg, R. Morandotti, Y. Silberberg, J. M. Arnold, G. Pennelli, and J. S. Aitchison, ibid. 19, 2938 (2002).

[4] A. Trombettoni, and A. Smerzi, Phys. Rev. Lett. 86, 2353 (2001); F. Kh. Abdullaev, B. B. Baizakov, S. A. Darmanyan, V. V. Konotop, and M. Salerno, Phys. Rev. A 64, 043606 (2001); G. L. Alfimov, P. G. Kevrekidis, V. V. Konotop, and M. Salerno, Phys. Rev. E 66, 046608 (2002); A. Smerzi and A. Trombettoni, Phys. Rev. A 68, 023613 (2003); Chaos 13, 766 (2003); N. K. Efremidis and D. N. Christodoulides, Phys. Rev. A 67, 063608 (2003).

[5] B. Malomed and M. I. Weinstein, Phys. Lett. A 220, 91 (1996).

[6] D. B. Duncan, J. C. Eilbeck, H. Feddersen, and J. A. D. Wattis, Physica D 68, 1 (1993); S. Flach, Y. Zolotaryuk, and K. Kladko, Phys. Rev. E 59, 6105 (1999); M. J. Ablowitz, Z. H. Musslimani, and G. Biondini, ibid. 65, 026602 (2002).

[7] I. E. Papacharalampous, P. G. Kevrekidis, B. A. Malomed, and D. J. Frantzeskakis, Phys. Rev. E 68, 046604 (2003).

[8] Yu. S. Kivshar, and B. A. Malomed, Rev. Mod. Phys. 61, 763 (1989); Y. S. Kivshar and D. K. Campbell, Phys. Rev. E 48, 3077 (1993); L. Brizhik, A. Eremko, L. Cruzeiro-Hansson, and Y. Olkhovska, Phys. Rev. B 61, 1129 (2000); P. G. Kevrekidis,
I. G. Kevrekidis, A. R. Bishop, and E. S. Titi, Phys. Rev. E 65, 046613 (2002).

[9] F. Kh. Abdullaev, E. N. Tsoy, B. A. Malomed, and R. A. Kraenkel, Phys. Rev. A 68, 053606 (2003).

[10] P. G. Kevrekidis, G. Theocharis, D. J. Frantzeskakis, and B. A. Malomed, Phys. Rev. Lett. 90, 230401 (2003).

[11] D. Anderson, Phys. Rev. A 27, 3135 (1983).

[12] M. Peyrard and M. D. Kruskal, Physica D 14, 88 (1984).

[13] B. A. Malomed, Phys. Rev. A 45, 4097 (1992); L. L. Bonilla and B. A. Malomed, Phys. Rev. B 43, 11539 (1991); T. Kuusela, J. Hietarinta, and B. A. Malomed, J. Phys. A 26, L21 (1993); G. Filatrella and B. A. Malomed, J. Phys.: Condens. Matter 11, 7103 (1999).

[14] T. Kuusela, Chaos, Solitons Fractals 5, 2419 (1995).

[15] G. Filatrella, B. A. Malomed, and R. D. Parmentier, Phys. Lett. A 198, 43 (1995).

[16] A. V. Ustinov and B. A. Malomed, Phys. Rev. B 64, 020302(R) (2001).

[17] H. E. Nistazakis, P. G. Kevrekidis, B. A. Malomed, D. J. Frantzeskakis, and A. R. Bishop, Phys. Rev. E 66, 015601(R) (2002).

[18] A. V. Ustinov, C. Coqui, A. Kemp, Y. Zolotaryuk, and M. Salerno, Phys. Rev. Lett. 93, 087001 (2004).

[19] B. Eiermann, Th. Anker, M. Albiez, M. Taglieber, P. Treutlein, K.-P. Marzlin, and M. K. Oberthaler, Phys. Rev. Lett. 92, 230401 (2004).

[20] Th. Anker, M. Albiez, R. Gati, S. Hunsmann, B. Eiermann, A. Trombettoni, and M. K. Oberthaler, Phys. Rev. Lett. 94, 020403 (2005). 